\title{
$\Lambda$ Cold Dark Matter, Stellar Feedback, and the Galactic Halo Abundance Pattern
}

\section{Citation}

Robertson, Brant, James S. Bullock, Andreea S. Font, Kathryn V. Johnston, and Lars Hernquist. 2005. " $\wedge$ Cold Dark Matter, Stellar Feedback, and the Galactic Halo Abundance Pattern." The Astrophysical Journal 632 (2): 872-81. https://doi.org/10.1086/452619.

\section{Permanent link}

http://nrs.harvard.edu/urn-3:HUL.InstRepos:41381703

\section{Terms of Use}

This article was downloaded from Harvard University's DASH repository, and is made available under the terms and conditions applicable to Other Posted Material, as set forth at http:// nrs.harvard.edu/urn-3:HUL.InstRepos:dash.current.terms-of-use\#LAA

\section{Share Your Story}

The Harvard community has made this article openly available.

Please share how this access benefits you. Submit a story.

Accessibility 
DRAFT VERSION OCTOBER 9, 2018

Preprint typeset using $\mathrm{LT}_{\mathrm{E}} \mathrm{X}$ style emulateapj v. 9/08/03

\title{
$\Lambda$ COLD DARK MATTER, STELLAR FEEDBACK AND THE GALACTIC HALO ABUNDANCE PATTERN
}

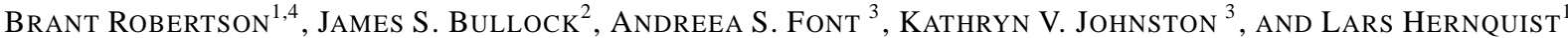 \\ Draft version October 9, 2018
}

\begin{abstract}
The hierarchical formation scenario for the stellar halo requires the accretion and disruption of dwarf galaxies, yet low-metallicity halo stars are enriched in $\alpha$-elements compared to similar, low-metallicity stars in dwarf spheroidal (dSph) galaxies. We address this primary challenge for the hierarchical formation scenario for the stellar halo by combining chemical evolution modelling with cosmologically-motivated mass accretion histories for the Milky Way dark halo and its satellites. We demonstrate that stellar halo and dwarf galaxy abundance patterns can be explained naturally within the $\Lambda$ CDM framework. Our solution relies fundamentally on the $\Lambda \mathrm{CDM}$ model prediction that the majority of the stars in the stellar halo were formed within a few relatively massive, $\sim 5 \times 10^{10} M_{\odot}$, dwarf irregular (dIrr) - size dark matter halos, which were accreted and destroyed $\sim 10 \mathrm{Gyr}$ in the past. These systems necessarily have short-lived, rapid star formation histories, are enriched primarily by Type II supernovae, and host stars with enhanced $[\alpha / \mathrm{Fe}]$ abundances. In contrast, dwarf spheroidal galaxies exist within low-mass dark matter hosts of $\sim 10^{9} M_{\odot}$, where supernovae winds are important in setting the intermediate $[\alpha / \mathrm{Fe}]$ ratios observed. Our model includes enrichment from Type Ia and Type II supernovae as well as stellar winds, and includes a physically-motivated supernovae feedback prescription calibrated to reproduce the local dwarf galaxy stellar mass - metallicity relation. We use representative examples of the type of dark matter halos we expect to host a destroyed "stellar halo progenitor" dwarf, a surviving dIrr, and a surviving dSph galaxy, and show that their derived abundance patterns, stellar masses, and gas masses are consistent with those observed for each type of system. Our model also self-consistently reproduces the observed stellar mass $-v_{\text {circ }}$ relation for local group satellites and produces the correct cumulative mass for the Milky Way stellar halo. We predict that the lowest metallicity stars in intermediate-mass dIrr galaxies like the SMC and LMC should follow abundance patterns similar to that observed in the stellar halo. Searches for accreted, disrupted, low-mass dwarfs may be enhanced by searching for unbound stars with dSph-like chemical abundance patterns.

Subject headings: galaxies: formation - galaxies: evolution - Galaxy: halo - Galaxy: abundances - galaxies: dwarf
\end{abstract}

\section{INTRODUCTION}

While evidence in support of a $\Lambda \mathrm{CDM}$ universe continues to mount from high-precision, large-scale cosmological observations (e.g. Spergel et al. 2003; Blakeslee et al. 2003; Pen et al. 2003; Lahav et al. 2002; Zehavi et al.|2002), a variety of data on galactic and subgalactic scales has remained more difficult to explain (e.g. Simon et al. 2005; Moore et al. 1999; Klypin et al. 1999; Navarro \& Steinmetz 1997, and references therein). Among the biggest questions in cosmology is whether $\Lambda$ CDM needs to be modified substantially in order to account for galactic-scale data (e.g. Spergel \& Steinhardt 2000; Kaplinghat et al. 2000; Colín et al. 2000) or if physical and dynamical processes can explain the data in the context of the standard paradigm (e.g. Bullock et al. 2000; Alam et al. 2002; Robertson et al. 2004). The resolution of this question requires testing our cosmological model against the full realm of existing subgalactic data. In this paper, we confront the rich data set of elemental abundances in galactic halo and Local Group dwarf galaxy stars with models set within the $\Lambda$ CDM cosmology. We conclude that the chemical abundance pattern in dwarf irregulars, dwarf spheroidals, and the Milky Way

\footnotetext{
${ }^{1}$ Harvard-Smithsonian Center for Astrophysics, 60 Garden St., Cambridge, MA 02138, USA

2 Department of Physics \& Astronomy, University of California, Irvine, CA 92697, USA

3 Van Vleck Observatory, Wesleyan University, Middletown, CT 06459 , USA

${ }^{4}$ brobertson@cfa.harvard.edu
}

stellar halo can be explained naturally in the context of our hierarchical cosmology.

One of the fundamental tenets of the CDM paradigm is that dark matter halos form hierarchically, via a series of mergers with smaller halos. This prediction gives rise to the natural expectation that the stellar halo is formed from disrupted, accreted dwarf galaxies (Johnston et al. 1996; Helmi \& White 1999; Bullock et al. 2001; Bullock \& Johnston 2005; Font et al. 2005. in preparation). Indeed, this picture is remarkably similar to the ideas of Searle \& Zinn (1978) who argued for "chaotic accretion" based purely on observational grounds. The discovery of fossil evidence of these accretion events via the identification of substructure within the stellar halo of our Galaxy may provide the only direct evidence that structure formation is hierarchical on small-scales (Bullock \& Johnston 2005). Searches for this substructure are under way (e.g. Newberg et al. 2002; Morrison et al. 2003; Kinman et al. 2004; Brown et al. 2004; Maiewski et al. 2004).

One of the main problems facing the hierarchical accretion picture for the stellar halo is that abundance patterns of surviving local group galaxies do not match those of stars in the stellar halo (see e.g. Wyse 2004; Venn et al. 2004, \$6). Stars in the stellar halo tend to be old, metal-poor, and $\alpha$ enriched, with $[\alpha / \mathrm{Fe}] \sim+0.3$. The stellar halo abundance pattern contrasts with the younger stellar populations of local, massive dwarf irregular (dIrr) galaxies like the Large Magellanic Cloud (LMC) and Small Magellanic Cloud (SMC), 
which are more metal enriched and have near-solar $[\alpha / \mathrm{Fe}] \mathrm{ra}-$ tios (Jasniewicz \& Thevenin 1994; Hill et al.1995; Hill 1997; Hill et al. 1997; Venn 1999; Cole et al. 2000; Hill et al. 2000; Korn et al. 2002; Smith 2002). Additionally, stars in dwarf spheroidal (dSph) galaxies around the Milky Way have intermediate $\alpha$-element abundances at low-metallicity, $[\alpha / \mathrm{Fe}] \sim$ +0.15 (Smecker-Hane \& McWilliam 1999; Shetrone et al. 2001; Smecker-Hane \& McWilliam 2003; Shetrone et al. 2003; Venn et al. 2004; Geisler et al. 2005). The discrepancy between the ages and metal abundances of dwarfs and the stellar halo is often cited as evidence against the hierarchical $\Lambda$ CDM prediction (e.g. Unavane et al. 1996; Gilmore \& Wvse 1998; Fulbright 2002; Tolstov et al. 2003; Venn et al. 2004), and has even been used to argue for a revision of the $\Lambda$ CDM cosmology (Wyse 2004). The problem is clear: how can one build the stellar halo from destroyed dwarf galaxies when the stars in low-mass galaxies appear to be chemically distinct from the stars in the halo?

Our solution to this conundrum relies fundamentally on two predictions that follow directly from the $\Lambda$ CDM cosmology: 1) most of the mass in the Milky Way halo was acquired via mergers with massive $\left(\sim 5 \times 10^{10} M_{\odot}\right)$ "dIrr-type" dark matter halos; and 2) most of these events occurred quite early on, at a look-back time of $\sim 10$ Gyr. It follows naturally that the majority of stars in the stellar halo were formed in intermediatemass halos of this type (Bullock \& Johnston 2005). The associated galaxies were disrupted soon after accretion and before significant chemical evolution.

Within the context of our scenario, the observed tendency for halo stars to be metal poor and $\alpha$-enhanced compared to stars in surviving dIrr galaxies follows from standard assumptions about Type Ia vs. Type II supernovae enrichment. Type II supernovae provide $\alpha$-enhanced enrichment within $\sim 10$ Myr of a star-formation episode (see $\$ 4$, while the iron-rich nucleosynthetic products from Type Ia supernovae contribute much later, at $\sim 1$ Gyr. The massive, "stellar halo progenitor" halos are accreted early and destroyed soon after. These systems are forced to have short, truncated star formation histories, and naturally host stars that are predominantly Type-II enriched and $\alpha$-enhanced. In contrast, surviving dIrr galaxies are accreted much later than their destroyed counterparts (this is what allows them to survive, see $\$ 2$, form stars for many billions of years, and have substantial enrichment from both Type Ia and Type II supernovae. Finally, lower-mass, dwarf spheroidal galaxies inhabit halos with much shallower potential wells $\left(\lesssim 10^{9} M_{\odot}\right)$ than the massive dIrr galaxies. We argue below that blow-out feedback from supernova give rise to both the low-metallicities observed in these systems as well as their intermediate $[\alpha / \mathrm{Fe}]$ ratios.

In what follows, we develop a model to track the chemical enrichment histories in dwarf galaxies forming in cosmologically self-consistent dark matter halos. We use three example mass accretion histories. Two are chosen to be representative of the kind of dark matter halos we expect to host surviving dSph and dIrr satellites, based on $\Lambda$ CDM predictions. A third example accretion history is typical of the type that is accreted and destroyed early to contribute to the formation of the halo. We track chemical enrichment by including contributions from Type Ia and Type II supernovae as well as stellar winds. Our star formation and feedback model selfconsistently reproduces: 1) the total mass of the stellar halo (Bullock \& Johnston 2005); 2) the stellar mass- $v_{\text {circ }}$ relation expected for Local Group dwarfs; and 3) the mass - metallicity relation found by Dekel \& Woo (2003) (see \$5). We go on to use this model to interpret the chemical abundance patterns of the stellar halo, dwarf spheroidals, and dwarf irregular galaxies (Venn et al. 2004).

We note that there exists an extensive literature of galactic chemical evolution modelling (van den Bergh 1962; Larson 1972; Tinsley 1972; Talbot \& Arnett 1973; Pagel \& Patchett 1975; Hartwick 1976; Tinslev 1980; Twarog 1980; Matteucci \& Greggio 1986; Matteucci \& Tornambe 1987; Matteucci \& Francois 1989; Wheeler et al. 1989; Ferrini et al. 1992; Timmes et al. 1995; Tsuiimoto et al. 1995; Devriendt et al. 1999; Ferreras \& Silk 2000; Chiappini et al. 2003; Lanfranchi \& Matteucci 2004). We aim neither to critique nor supersede this previous work. Our goal is simply to approach galactic chemical evolution with a hierarchically driven and cosmologically consistent method while retaining complexity in our chemical enrichment code (see also Brook et al. 2004a b). By placing the chemical enrichment of dwarf galaxies into the context of their expected cosmological formation and subsequent fate, we hope to gain some insight on interpreting the rich data sets being compiled from Local Group observations.

In the next section, we provide an overview of the expected formation histories of Milky Way-type dark matter halos, the accretion and disruption histories of their accreted subhalos, and the mass accretion histories of the satellite halos themselves. In $\$ 3$ we discuss our star formation law and present our three example mass accretion histories used to facilitate our comparisons to dIrr, dSph, and stellar halo abundance data. We detail our stellar yield modelling in $\$ 4$ and our chemical evolution and feedback calibration in $\$ 5$ We present our results in $\$$ and summarize and conclude in $\$ 7$ Throughout this paper we assume a flat $\Lambda$ CDM cosmology with $\Omega_{m}=0.3, \Omega_{\Lambda}=0.7, h=0.7$, and $\sigma_{8}=0.9$.

\section{COSMOLOGICAL CONTEXT}

We first review several relevant expectations for the formation of galaxy-size dark matter halos within the $\Lambda \mathrm{CDM}$ framework. The reader is referred to Wechsler et al. (2002); Zentner \& Bullock (2003) and Zentner et al. (2005) for more detailed discussions.

\subsection{Hierarchical growth of the Milky Way dark halo}

Consider a galaxy-size dark matter halo of mass $M_{\mathrm{h}}$ at $z=0$. Mass growth in halos of this type proceeds by the merging and accretion of a wide mass spectrum of "subhalos" or "satellite halos". Integrated over the lifetime of the host halo, the accreted spectrum of satellite halo masses, $M_{s}$, rises softly towards the low-mass end, $d N / d M_{\mathrm{s}} \propto M_{\mathrm{s}}^{-1 / 2}$ and falls off sharply above a characteristic cutoff mass $\sim 0.1 M_{\mathrm{h}}$. Owing to the shallow slope, most of the mass accreted onto the host is associated with subhalos of roughly the cutoff mass.

The Milky Way's dark matter halo has a virial mass of $M_{\mathrm{h}} \simeq 10^{12} M_{\odot}$ at $z=0$ and is thus expected to have accreted most of its mass via the accretion of intermediate size systems $M_{\mathrm{s}} \simeq(1-10) \times 10^{10} M_{\odot}$ (Zentner \& Bullock 2003). Accreted subhalos lose mass as a result of violent interactions with the host halo system, and are typically destroyed within a few dynamical times. The stripped material incorporates into and effectively builds the background dark matter halo of the host. The model of Bullock \& Johnston (2005) that serves as our cosmological framework accounts for these dynamical effects, in addition to the redshift evolution of the characteristic mass and densities of merging systems. 
The time line of accretion activity is sensitive to the host halo mass and the governing cosmological parameters. For Milky-Way size halos in the standard $\Lambda$ CDM model, the accretion rate of dwarf-galaxy halos peaks $\sim 10 \mathrm{Gyr}$ in the past, and slows down considerably at a look-back time of $\sim 8 \mathrm{Gyr}$, although there is scatter from halo to halo (Zentner \& Bullock 2003). Satellite halos that get accreted during the earliest epochs have time to be destroyed by interactions with the host galaxy's environment (see Zentner et al. 2005). The increased efficiency of dynamical friction with satellite mass causes more massive subhalos to lose mass more quickly than smaller subhalos. Most of the subhalos that survive until the present day were accreted during the slow-growth phase, within the past $\sim 5 \mathrm{Gyr}$, and therefore represent a temporally biased population compared to their destroyed counterparts. We emphasize that this temporal bias between surviving and destroyed systems implies that the two populations have intrinsically different formation histories: the destroyed population of halos are expected to have formed more rapidly than the subhalos that survive.

We expect that the most massive, accreted satellite halos will host dwarf galaxies, and that most of these systems will be destroyed by tidal interactions. Just as the dissipationless dark halo of the host is built from the dark matter that was originally associated with accreted and destroyed subhalos, the stellar halo is built from the dissipationless stellar matter that was associated with the same population of destroyed galaxies (Bullock et al. 2001). Given this scenario for stellar halo formation, two important implications follow directly from the $\Lambda \mathrm{CDM}$ predictions discussed above:

1. The majority of the stars contained in the Milky Way stellar halo originated in $\sim 5 \times 10^{10} M_{\odot}$ " dwarf irregular-type" dark matter halos.

2. The surviving satellite galaxies around the Milky Way represent a biased population: they typically were accreted later than the dwarf galaxies that were destroyed to make the halo.

The second point implies that the mass accretion and star formation histories of surviving satellites are more gradual than their destroyed counterparts.

\subsection{Dark matter halo mass accretion histories}

Wechsler et al. (2002) used N-body simulations to study the mass accretion histories of dark matter halos observed at various redshifts. One of the main results of their work was to show that the cumulative mass accretion in individual dark matter halos can be well-described by a simple function:

$$
M(a)=M_{0} \exp \left[-2 a_{c}\left(\frac{a_{0}}{a}-1\right)\right],
$$

where $a=(1+z)^{-1}$ is the expansion factor and $M_{0}$ is the mass of the halo as observed at the epoch $a_{0}=\left(1+z_{0}\right)^{-1}$. Given a halo mass and redshift, this function has only one parameter, $a_{c}$, which is a characteristic formation epoch for the halo. The typical value of $a_{c}$ varies as a function of halo mass, with more massive halos having earlier formation times. At a fixed $M_{0}$, the distribution of $a_{c}$ is roughly log-normal, with a scatter of $\Delta \log _{10} a_{c}=0.14$. Of note is the interesting fact that the $a_{c}$ distribution is independent of $a_{0}$ for halos of a fixed mass.

Wechsler et al. (2002) showed that the median value of the formation epoch parameter, $a_{c}$, can be determined for a halo of mass $M_{0}$ using a simple model based on spherical collapse. Specifically, $a_{c}$ is set by the epoch at which $M_{*}$, the typical collapsing mass (e.g. Lacey \& Cole 1993), equals a fixed fraction $F=0.015$ of the halo mass $M_{0}$ :

$$
M_{*}\left(a_{c}\right) \equiv F M_{0} .
$$

As expected, this expression for $a_{c}$ is independent of $a_{0}$. A Milky-Way type halo, for example, has a median $a_{c} \simeq 0.30$, corresponding to a look-back time of $\sim 10.5 \mathrm{Gyr}$, which is consistent with the expected epoch of peak accretion discussed above.

\section{STAR FORMATION AND EXAMPLE HISTORIES}

We will use three example mass accretion histories to explore expected differences between halo stars and surviving satellites. Since each system under consideration is a lowmass object with efficient gas cooling, we assume the cold gas inflow rate, $g(t)$, tracks the dark matter accretion rate:

$$
g(t)=f_{\text {gas }} h\left(t-t_{\text {in }}\right) .
$$

Here, $f_{\text {gas }}$ is the fraction of the dark matter halo mass in the form of cold, accreting gas, and $h(t)=\mathrm{d} M / \mathrm{d} t$ is the halo growth rate determined by taking the derivative of Equation (1) with respect to cosmic time. The time lag $t-t_{\text {in }}$ between $h(t)$ and $\left.g_{(} t\right)$ accounts for the finite time it takes for gas to settle into the central galaxy after being accreted onto the halo. We assume this settling occurs in roughly a dynamical time at the virial radius: $t_{\text {in }}=R_{\mathrm{h}} / v_{\text {circ }} \simeq 2 \mathrm{Gyr}(1+z)^{-3 / 2}$. We set $f_{\text {gas }}=0.02$, in accord with the cold baryonic mass fraction in observed galaxies (Bell et al. 2003).

The star formation rate, $\psi(t)$, in each system is set using the simple relation

$$
\psi(t)=\frac{M_{\mathrm{gas}}}{\tau_{\star}},
$$

where $M_{\text {gas }}$ is the total cold gas mass in the system and the star formation timescale, $\tau_{\star}$, is a free parameter. In the models we explore below we assume for simplicity that star formation is truncated soon after each satellite halo is accreted onto the Milky Way host as a result of ram-pressure stripping from the background hot gas halo (Moore \& Davis 1994; Maller \& Bullock 2004). Of course, this assumption is oversimplified and cannot be accurate in detail, but it allows us to capture the general differences we expect from one example to the next without addressing the complicated interplay between orbital evolution and star formation. Ram-pressure will be more effective at stripping gas from low-mass objects with shallow potential wells (dSph) while realistically dIrrsize halos will tend to retain their gas more efficiently. Additionally, for a given mass, typical encounter velocities are higher at higher redshifts. Surviving dwarf satellites will undergo correspondingly less ram-pressure stripping than their counterparts that are accreted into the halo early and subsequently disrupted. However, for massive systems that were accreted early (a "halo-progenitor", for instance) our assumption of star formation truncation will not qualitatively affect our results compared to a model where star formation is allowed to continue until the galaxy is tidally destroyed. Earlyaccreted systems are typically destroyed very soon after they merge (over $\mathrm{a} \sim 1$ Gyr timescale, a typical dynamical time for a $\mathrm{Z} \sim 1$ halo). Nevertheless, we acknowledge our assumption of truncated star formation is simplified. We aim to explore the implications of varying models of star formation within satellite halos in subsequent work. 
The gas mass in Equation 4 will not simply be the cumulative integral of gas inflow rate, $g(t)$, as we allow for the effect of star formation feedback and associated gas loss within each satellite halo. The system of equations we use to track star formation, gas loss, and metal enrichment is presented in $\$ 4$. Including this gas outflow, we find that the choice $\tau_{\star}=6.75$ Gyr allows us to match the observed mass-to-light ratios in dIrr-sized objects, and, when incorporated into our full mass accretion history models of the Milky Way, the same choice gives a good match to the total Milky Way stellar halo mass as well as its radial profile (Bullock \& Johnston 2005). The inclusion of feedback in our model helps regulate the star formation rate in dwarf galaxies. As a result, we must adopt a smaller star formation timescale than that required in the similar model utilized by Bullock \& Johnston (2005) which does not explicitly use a prescription for feedback from star formation. Before moving on to discuss our treatment of outflow and metal enrichment in detail, we first introduce our three example mass accretion histories.

\section{Dwarf Irregular Satellite}

For our surviving, "dIrr" satellite halo example, we use a dark matter halo of virial mass of $M_{0}=6 \times 10^{10} M_{\odot}$, which we assume is accreted into the Milky Way halo at $a_{0}=0.79$. This accretion epoch corresponds to a look-back time of 3.1 Gyr, a typical accretion time for surviving, massive satellites (A. Zenter \& J. Bullock, private communication) and close to that expected for the LMC from gravitational and hydrodynamical simulations of its orbital evolution within the Milky Way potential (Mastropietro et al. 2004). A halo of this mass at $a_{0}=0.79$ has a median maximum circular velocity of $v_{\text {circ }}=72 \mathrm{~km} \mathrm{~s}^{-1}$ (Bullock et al. 2001), but we expect that dark matter mass loss will leave this system with a circular velocity closer to that of the SMC or LMC $\left(\sim 60 \mathrm{~km} \mathrm{~s}^{-1}\right.$; van der Marel et al. 2002) after three billion years of evolution (Bullock \& Johnston 2005).

We assume that the dark matter mass accretion history of our dIrr halo follows the form of Equation 1, with a characteristic formation epoch of $a_{c}=0.231$ (Equation 2), corresponding to a look-back time of $11.6 \mathrm{Gyr}$. Note that the time between "formation" at $a_{c}$ and its accretion into the Milky Way dark halo at $a_{0}$, this system will have had $\sim 8.5$ Gyr to form stars - ample time for both Type Ia and Type II enrichment. After applying the star formation law discussed above and the feedback prescription described in $\S 4$, the final stellar mass and gas mass in this system are $M_{\star}=3.9 \times 10^{8} M_{\odot}$ and $M_{\text {gas }}=4.7 \times 10^{8} M_{\odot}$, respectively. Allowing for a small amount of gas loss from ram pressure stripping after accretion (see e.g. Mastropietro et al. 2004), these numbers are in line with gas mass and stellar mass measurements for the SMC and LMC (e.g. Grebel 2001; Grebel et al. 2003).

\section{Dwarf Spheroidal Satellite}

Our Dwarf Spheroidal example consists of a relatively lowmass halo, $M_{0}=5.6 \times 10^{8} M_{\odot}$, accreted at $a_{0}=0.667$ or at a look-back time of $5 \mathrm{Gyr}$. The accretion time is typical for that of surviving satellite halos of this size (A. Zentner \& J. Bullock, private communication). The halo's formation epoch is $a_{c}=0.08$, corresponding to a look-back time of $13.1 \mathrm{Gyr}$. This system has a maximum circular velocity of $v_{\text {circ }}=20 \mathrm{~km} \mathrm{~s}^{-1}$ at accretion, which will likely be reduced to $v_{\text {circ }} \simeq 15 \mathrm{~km} \mathrm{~s}^{-1}$ after five billion years of evolution within the galaxy potential (Bullock \& Johnston 2005). This circular velocity agrees with circular velocity estimates for $\mathrm{dSph}$ satellite galaxies in the Local Group (e.g. Zentner \& Bullock 2003). The time available for star formation between $a_{c}$ and $a_{0}$ is $\sim 8 \mathrm{Gyr}$ for this system.

It is now well-known that if $\Lambda \mathrm{CDM}$ is correct, only roughly one-in-ten of the dwarf-size satellite halos can host a dwarf galaxy, with the rest remaining dark (Moore et al. 1999; Klypin et al. 1999). A natural solution to this problem relies on the fact that halos of this size $\left(v_{\text {circ }} \lesssim 30 \mathrm{~km} \mathrm{~s}^{-1}\right)$ are unable to accrete gas after the universe becomes reionized (e.g. Bullock et al. 2000). We account for this effect by setting the gas accretion rate $g(t)=0.0$ after the epoch of reionization, $a_{\text {re }}$. The value of $a_{c}$ we have adopted for our dSph example is $\sim 2 \sigma$ earlier than the median expected for a halo of its size (via Equation 2). We have made this choice in order to ensure that this halo collapses before cosmic reionization and is able to host a visible dwarf galaxy. Our assumption about the effect of reionization on dwarf galaxy formation allows our picture to self-consistently address the "dwarf satellite problem" without modifying the tenets of $\Lambda \mathrm{CDM}$. For concreteness, we choose $a_{\mathrm{re}}=0.09$, but our results are insensitive to this choice.

The stellar mass and gas mass within this system at accretion is $M_{\star}=1.1 \times 10^{6} M_{\odot}$ and $M_{\text {gas }}=3.8 \times 10^{5} M_{\odot}$ respectively. The stellar mass is consistent with that of $\mathrm{dSph}$ galaxies in the local group, and its gas mass fraction is lower than that in the dIrr example, also in accordance with observed trends (e.g. Mateo 1998; Grebel et al. 2003, and references therein). We expect that the gas mass in this system will be further reduced after accretion as a result of efficient ram pressure stripping from the shallow host halo potential well.

\section{Stellar Halo Progenitor}

As argued in \$2.1, and discussed in more detail in Bullock \& Johnston (2005), the hierarchical scenario predicts that most of the mass in the stellar halo was contributed by several massive subhalos that were accreted onto the Milky Way host long ago. We choose to model one such subhalo as our example "stellar halo progenitor" a dark halo with a virial mass equal to that of our dIrr example above, $M_{0}=6 \times 10^{10} M_{\odot}$, but which was accreted $9 \mathrm{Gyr}$ in the past, at $a_{0}=0.417$. Because this subhalo has the same mass as the dIrr example, the stellar halo progenitor has the same formation epoch, $a_{c}=0.231$. However, because the subhalo was accreted (and destroyed) at a much earlier time, the time available for star formation in this system is only $\sim 2.6 \mathrm{Gyr}$, and we therefore expect that the $\sim 1$ Gyr required for Type Ia enrichment will limit significant Type Ia enrichment in this system.

When we apply our star formation and feedback model, the stellar mass at accretion in this system is $M_{\star}=2.4 \times 10^{8} M_{\odot}$ and the cold gas mass is $M_{\text {gas }}=7.2 \times 10^{8} M_{\odot}$. The high gas fraction is a result of have a shorter time available to turn gas into stars. Indeed, since a majority of the baryons in the Milky Way system were accreted in early events of this size (\$2.1), gas-rich mergers of this type are likely fundamental in fueling the growing Milky Way bulge and disk components. Although a large number of lower-mass subhalo accretion events will likely contribute significant substructure to the Galactic halo, we expect that $\sim 4-5$ mergers of subhalos similar to our halo progenitor model are responsible for most of the mass of the Milky Way stellar halo.

In Figure 1 we plot star formation histories as a function 


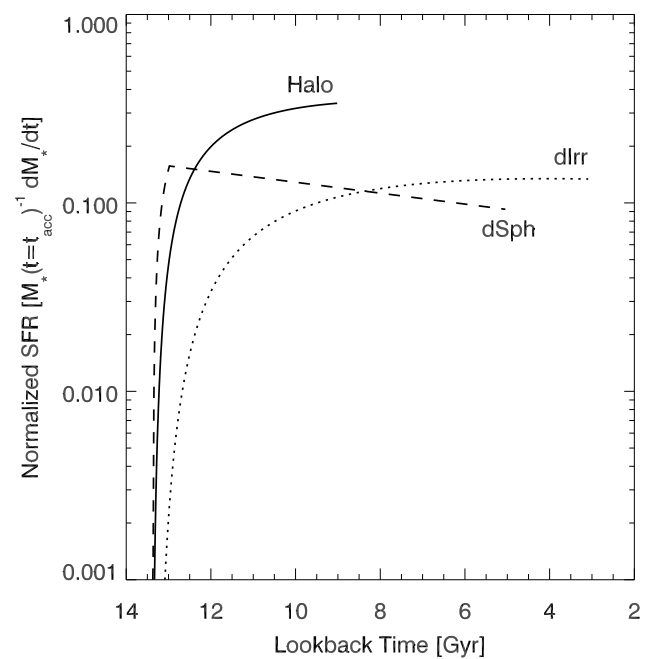

FIG. 1.- Model star formation histories of our halo-progenitor example (solid line), dIrr (dotted line), and low-mass dSph galaxy (dashed line). The halo-progenitor model forms stars quickly until it is accreted into the stellar halo $\sim 9$ Gyr ago. The surviving dIrr galaxy forms stars more gradually, until its recent accretion into the galaxy $\sim 3$ Gyr ago. The dwarf spheroidal galaxy has its gas infall suppressed by reionization early-on and converts its gas mass into stars until its accretion into the galaxy $\sim 5 \mathrm{Gyr}$ ago. Both the dIrr and dSph are expected to survive as Milky Way satellites until the present day.

of look-back time for each example history: halo-progenitor (solid line), dwarf irregular (dotted line), and dwarf spheroidal (dashed line). The break in the dSph track at $\sim 13$ Gyr is associated with suppression of gas infall at reionization. From Figure 1 we see that the halo-progenitor star formation history is truncated and more rapid compared with the surviving dIrr. The history of the $\mathrm{dSph}$ example is intermediate between the two. We now address how these differences will manifest themselves as chemical signatures in the different stellar systems.

\section{STELLAR YIELDS}

In order to track the time-dependent chemical content of dwarf galaxies during their formation, we model the chemical yields from stellar populations, including Type Ia supernovae, Type II supernovae, and stellar winds from intermediate mass stars. The rate of enrichment is calculated analytically and the yields for individual chemicals are selected from the literature.

\subsection{Initial Mass Function}

We define our initial mass function (IMF) as

$$
\phi(m) \equiv \frac{\mathrm{d} N}{\mathrm{~d} m}=B\left(m / M_{\odot}\right)^{-s},
$$

which provides the differential number of stars of mass $m$. We adopt the Kroupa (2001, 2002) IMF, which has a massdependent slope $s$ given by

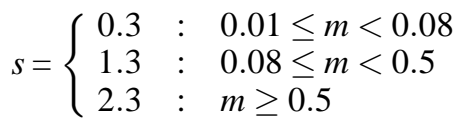

We set the constant $B$ to normalize the IMF to give

$$
\int_{0.1}^{100} m \phi(m) \mathrm{d} m=1 M_{\odot},
$$

as we consider stars of mass $0.1 \leq m \leq 100$. Our normalization provides $\phi(m)$ in units of $\overline{M_{\odot}^{-1}}$ and allows us to calculate our yields per unit solar mass of stars. We note that for stellar masses $m>0.5 M_{\odot}$ the Kroupa IMF is very similar to the Salpeter (1955) IMF that has a constant slope of $s=2.35$, though, as discussed by Kroupa (2002), uncertainties in determining the IMF of massive stars may mask a steeper slope of $s \approx 2.7$ for stars above $1 M_{\odot}$.

\subsection{Type II Supernovae}

Given an IMF, we can track the cumulative number of Type II supernovae that have occurred per solar mass of star formation as a function of time $t$ as

$$
N_{I I}(<t)=\int_{\mathcal{M}_{\star}(t)>\mathcal{M}_{I I, \min }}^{\mathcal{M}_{I I, \max }} \phi(m) \mathrm{d} m .
$$

Here, $\mathcal{M}_{\star}(t)$ is the mass of a star with stellar lifetime $t$ and $\mathcal{M}_{I I, \text { min }}$ is the minimum mass star producing a Type II supernova and $\mathcal{M}_{I I, \max }$ is the maximum stellar mass that will produce Type II yields. We assume that all stars more massive than $\mathcal{M}_{I I, \max }=50 M_{\odot}$ will collapse completely into black holes and we use $\mathcal{M}_{I I \text { min }}=10 M_{\odot}$.

For the stellar lifetime as a function of star mass, we adopt a dual power-law fit to the Schaller et al. (1992) stellar lifetimes of the form

$$
t_{\star}(\mathcal{M})=10.5\left(\frac{\mathcal{M}}{M_{\odot}}\right)^{-3}+0.035\left(\frac{\mathcal{M}}{M_{\odot}}\right)^{-0.55}
$$

with $t_{\star}$ in Gyr. The the inverse quantity $\mathcal{M}_{\star}(t)$ and its derivative $\mathrm{d} \mathcal{M}_{\star} / \mathrm{d} t_{\star}$ can be obtained from this relation. The rate of Type II supernovae per solar mass in each stellar population is

$$
R_{I I}(t)=-\frac{\mathrm{d} \mathcal{M}}{\mathrm{d} t_{\star}} \phi\left[\mathcal{M}_{\star}(t)\right],
$$

with no further normalization being necessary as all massive stars between $\mathcal{M}_{I I \text {,min }}$ and $\mathcal{M}_{I I \text {,max }}$ are considered to produce supernovae.

The instantaneous chemical yield of an element $X$ from Type II supernovae as a function of time after a stellar population forms will then follow

$$
Y_{X, I}(t)=y_{X, I I}[\mathcal{M}(t)] R_{I I}(t),
$$

where $y_{X, I I}[\mathcal{M}]$ is the Type II yield of element $X$ from a star of mass $\mathcal{M}$. In practice, we use the Type II yields from Tsuijimoto et al. (1995) and Thielemann et al. (1996), interpolating between their simulated models in order to obtain yields as a continuous function of mass.

Given the above model, Type II supernovae provide enrichment from $t_{\star}(50)=4.1$ Myr until $t_{\star}(10)=20.4$ Myr after each stellar population forms.

\subsection{Type Ia Supernovae}

In calculating the rate of Type Ia supernova, we follow the formalism developed by Greggio \& Renzini (1983), which postulates that the progenitors of Type Ia supernovae are binary star systems. The rate of Type Ia supernovae is set by the evolution of the secondary star in each binary system.

We define the number of Type Ia supernovae that have occurred before time $t$ as

$$
N_{I}(<t)=A \int_{\mathcal{M}_{b, \mathrm{inf}}}^{\mathcal{M}_{b, \max }} \phi\left(\mathcal{M}_{b}\right)\left[\int_{\mu_{\text {inf }}}^{1 / 2} 2^{1+\gamma}(1+\gamma) \mu^{\gamma} \mathrm{d} \mu\right] \mathrm{d} \mathcal{M}_{b}
$$


where $A$ is a normalization parameter. The outer integral sums over the total binary mass, $\mathcal{M}_{b}=\mathcal{M}_{1}+\mathcal{M}_{2}$, with $\mathcal{M}_{2}<\mathcal{M}_{1}$, and the term in brackets describes the distribution of secondary mass fractions $\mu=\mathcal{M}_{2} / \mathcal{M}_{b}$. The exponent $\gamma=2$ sets the spectrum of secondary masses. The upper limit on the total binary mass is set by twice the maximum primary mass that allows the development of a degenerate $\mathrm{C} / \mathrm{O}$ core before filling its Roche lobe, $\mathcal{M}_{b, \max }=16 M_{\odot}$. The lower limit forces $M_{2} \leq M_{1}$ via $\mathcal{M}_{b \text {.inf }}=\operatorname{MAX}\left[\mathcal{M}_{2}(t), \mathcal{M}_{b, m i n}\right]$ with $\mathcal{M}_{b, \min }=3 M_{\odot}$ following Greggio \& Renzini (1983). As in $\$ 3.2, \mathcal{M}_{2}(t)$ is the mass corresponding to the stellar lifetime $t$. The lower limit on the secondary mass fraction is $\mu_{\text {inf }}=\operatorname{MAX}\left[\mathcal{M}_{2}(t) / M_{b},\left(M_{b}-\frac{1}{2} M_{b, \max }\right) / M_{b}\right]$. In order to approximate local supernovae rates as determined by Tammann (1982), we follow the Matteucci \& Greggio (1986) Type I SN normalization. Adjusting the Matteucci \& Greggio (1986) result for our chosen IMF, we find $A \approx 0.0956$.

Given the cumulative number of Type Ia supernovae as a function of time $t$, the corresponding rate is simply

$$
R_{I}(t)=\frac{\mathrm{d} N_{I}(<t)}{\mathrm{d} t} .
$$

The instantaneous chemical yield for each element $X$ from Type Ia supernovae is then $Y_{X, I}(t)=y_{X I} R_{I}(t)$. For the individual yield per supernovae $y_{X, I}$, we adopt the yields of the Nomoto W7 Type Ia supernova model (Nomoto et al. 1984, 1997). In this model the Type Ia supernovae provide enrichment for $t>$ $t_{\star}(8)=31.7 \mathrm{Myr}$ after each stellar population forms, with the peak of the Type I rate occurring at $t \sim 1 \mathrm{Gyr}$.

\subsection{Stellar Winds}

We follow the chemical enrichment from stellar winds by tracking the death rate of stars and assuming the majority of the stellar mass loss occurs during asymptotic giant branch thermal pulsing near the end of the stellar lifetime. Our effective rate of stellar winds is then

$$
R_{W}(t)=-\frac{\mathrm{d} \mathcal{M}}{\mathrm{d} t_{\star}} \phi(\mathcal{M})
$$

which, like the Type II supernovae rate, is set by the IMF and stellar lifetimes. The instantaneous chemical yield of an element $X$ from stellar winds is then $Y_{Y W}(t)=y_{Y W} R_{W}(t)$. We utilize the van den Hoek \& Groenewegen (1997) timedependent stellar wind yields $y_{X, W}$, interpolating over mass and metallicity for stellar masses between $0.8-8 M_{\odot}$. Stellar winds provide enrichment in this model continuously after $t_{\star}(8)=31.7$ Myr.

\section{CHEMICAL ENRICHMENT AND FEEDBACK}

\subsection{Tracking Abundances}

We aim to track the abundances of $\mathrm{H}, \mathrm{He}, \mathrm{Fe}$, and the $\alpha$ elements $\mathrm{O}$ and $\mathrm{Mg}$ using the three example accretion histories discussed in $\$ 2$. To do so, we follow the evolution of stellar mass $M_{\star}$, gas mass $M_{\text {gas }}$, and mass $M_{X}$ in each element $X$ using the system of equations:

$$
\begin{aligned}
\frac{\mathrm{d} M_{\star}}{\mathrm{d} t} & =\psi(t)-Y(t) \\
\frac{\mathrm{d} M_{\mathrm{gas}}}{\mathrm{d} t} & =-\psi(t)+Y(t)+g(t)-e(t) \\
\frac{\mathrm{d} M_{X}}{\mathrm{~d} t} & =-f_{X} \psi(t)+Y_{X}(t)+h_{X} g(t)-f_{X} e_{X}(t) .
\end{aligned}
$$

As discussed in $\$ 2.3$, the star formation rate is assumed to track the mass in cold gas as $\psi(t)=M_{\text {gas }} / \tau_{\star}$, and the gas infall rate, $g(t)$, tracks the halo mass accretion rate. The quantity $Y(t)$ is the total rate of mass input into the gas from supernovae of both types and stellar winds, summed over all stellar populations. Similarly, $Y_{X}(t)$ is the instantaneous input rate of the specific element $X$, again summed over supernovae and winds for all stellar populations. The gas mass fraction in each element $X$ is $f_{X}=M_{X} / M_{\text {gas }}$, and $h_{X}$ is the elemental mass fraction in newly accreted gas. The ejection rate for each element is chosen to follow the star formation rate as $e_{X}(t)=w_{X} \psi(t)$, with $w_{X}$ representing the efficiency of gas blow-out for element $X$. The total gas ejection rate in the second equation is then the sum over all elements $X, e(t)=\Sigma f_{X} e_{X}(t)$.

Each star formation history begins by accreting low- metallicity, alpha-enriched gas, with $[\mathrm{Fe} / \mathrm{H}]=-4.0$ and $[\alpha / \mathrm{Fe}]=0.3$. Throughout the history of each galaxy, we assume that the infalling gas retains this initial Type II-enriched abundance pattern (this sets $h_{X}$ in Equation 17), although, in detail, our model results are insensitive to the abundance pattern of the low-metallicity infalling gas. In the results presented in \$6 we define our abundances relative to the solar abundances measured by Grevesse \& Sauval (1998). We turn now to a more in-depth discussion of our treatment of blow-out feedback. Our feedback prescription is important for explaining the abundance pattern in low-mass, dSph-type galaxies.

\subsection{Feedback and the Mass-Metallicity and Mass- $v_{\text {circ }}$ Relations}

Gas and metal ejection from supernovae winds almost certainly plays an important role in shaping the properties of galaxies - especially dwarf-sized galaxies (Dekel \& Silk 1986). Dekel \& Woo (2003) (DW) have used a compilation of dwarf galaxy data to show that the stellar masses and metallicities of dwarf galaxies follow a fairly tight relation, $Z \propto M_{\star}^{2 / 5}$, and have argued that blow-out feedback can naturally explain this trend. Although similar relations were noted previously (e.g., Brodie \& Huchra 1991; Zaritsky et al. 1994), we refer to this trend as the "Dekel-Woo relation", and use it to motivate and normalize our prescription for galactic supernovae ejecta. The feedback prescription we present below in fact reproduces the Dekel-Woo relation, but also gives rise to and explains the $[\alpha / \mathrm{Fe}]$ abundance pattern trends observed for lowmass dwarf galaxies. We note that our prescription for gas and metal ejection does not strongly affect our conclusions regarding the stellar halo progenitor abundance pattern nor those of our surviving dIrr example. The robustness of the model predictions for these subhalos results because the dark halos of these systems are relatively massive. However, ejection plays an important role in the star formation and enrichment history of our lower-mass dSph example, where the typical energy of supernovae ejecta is significant compared to the binding energy of the halo.

We can estimate the efficiency of gas and metallicity ejection from dwarf halos, $w$, by taking the ratio of specific energy of gas that escapes the central dwarf galaxy as a wind, $\mathcal{E}_{\mathrm{w}}$, to the specific binding energy of the halo:

$$
w \approx \frac{\mathcal{E}_{\mathrm{w}}}{\frac{1}{2} v_{\text {circ }}^{2}} \approx \frac{\epsilon \eta E_{\mathrm{SN}} N_{\mathrm{SN}}}{\frac{1}{2} v_{\text {circ }}^{2}},
$$

where $v_{\text {circ }}$ is the circular velocity of the halo in question. The energy of the gas that leaves the central galaxy will depend on $N_{\mathrm{SN}}$, the number of supernovae per unit mass in the stellar population and $E_{\mathrm{SN}}$, the energy per supernovae. The factor $\eta$ is the fraction of supernovae energy converted into kinetic 
energy, and $\epsilon$ is the fraction of the input kinetic energy that is retained by the gas that actually escapes the galaxy as a wind. The kinetic energy fraction $\eta$ will depend on the speed at which supernovae bubbles overlap compared to the radiative timescale of the gas in the galaxy, and therefore on the supernovae rate (Dekel \& Silk 1986). It typically will not exceed a few percent, and is commonly taken to be $5 \%$ in cosmological simulations of galaxy formation (c.f. Abadi et al. 2003). With this, typical numbers give $w \approx 22 \epsilon \eta_{5} v_{50}^{-2}$, where $\eta_{5} \equiv \eta / 0.05$ and $v_{50} \equiv v_{\text {circ }} / 50 \mathrm{~km} \mathrm{~s}^{-1}$, and we have used $N_{\mathrm{SN}} \approx 1.1 \times 10^{-2} M_{\odot}^{-1}$, which follows from our chosen IMF and stellar population modelling.

The fraction of kinetic energy $\epsilon$ retained by gas that escapes as a wind will vary between elements. While $\mathrm{H}$ and $\mathrm{He}$ will primarily not be entrained in the wind because of interactions with the surrounding ambient medium, $\epsilon \ll 1$, metals will be preferentially blown-out of galaxies in the metal-rich supernovae ejecta, $\epsilon \sim 1$ (Mac Low \& Ferrara 1999). Furthermore, the rate of Type Ia supernovae per stellar population is quite slow, dragging out over Gyr timescales, while the Type II supernovae rate is rapid. We therefore expect that the bubble overlap time and kinetic energy fraction to be slightly lower for Type Ia compared to Type II ejecta: $\eta_{\alpha} \lesssim \eta_{F e}$. Motivated by this physical picture, we set the gas blow-out efficiency of gas, $\mathrm{Fe}$, and $\alpha$-elements in halos of varying circular velocities to be

$$
\begin{aligned}
& w_{\text {gas }}\left(v_{\text {circ }}\right)=0.15 \times\left(\frac{v_{\text {circ }}}{50 \mathrm{~km} \mathrm{~s}^{-1}}\right)^{-2}, \\
& w_{\alpha}\left(v_{\text {circ }}\right)=14.4 \times\left(\frac{v_{\text {circ }}}{50 \mathrm{~km} \mathrm{~s}^{-1}}\right)^{-2}, \\
& w_{F e}\left(v_{\text {circ }}\right)=16.8 \times\left(\frac{v_{\text {circ }}}{50 \mathrm{~km} \mathrm{~s}^{-1}}\right)^{-2} .
\end{aligned}
$$

We have adopted the standard kinetic energy fraction $\eta=0.05$ for gas and $\mathrm{Fe}$, but a slightly lower value, $\eta=0.035$, for the $\alpha$ elements. We have used $\epsilon=0.01$ for the gas and $\epsilon=0.8$ for the metals.

While our choices for $w_{F e}$ and $w_{\text {gas }}$ are of roughly the expected size, we have chosen their precise values in order to reproduce the observed $M_{\star}-v_{\text {circ }}$ relation for dwarf galaxies in the local group (DW; see Bullock \& Johnston 2005) as well as to approximate the Dekel-Woo mass-metallicity relation. Figure 2 shows the stellar-mass metallicity relation estimated by DW as the solid line, with the dashed lines indicating the $1-\sigma$ scatter in metallicities about the relation. The two solid circles and the open circle show our model results for the dSph, dIrr, and stellar halo progenitor examples, respectively. The error bars reflect the $68 \%$ spread in metallicity range for stars in each model galaxy. In order to more fully compare with the observed relation, we have also included results for a range of surviving satellite halos from the models of Bullock \& Johnston (2005). The mass accretion histories of these satellites were generated in the same way discussed $\S 2$, except now we span a more complete range in $v_{\text {circ }}$. The model galaxies generally reproduce the observed trend, with good agreement considering the uncertainty in the data and the difficulty in assigning precise stellar masses to these observed systems. We retain the stellar feedback calibration that provides this agreement for the proceeding calculations.

Note that there is very little freedom in our parameter choices. Recall that $t_{\star}$ and $f_{\text {gas }}$ in our mass accretion model are already fixed to reproduce the stellar mass of the Galactic halo and the cold baryon fraction in galaxies. Once these are given, $w$ is constrained by the stellar mass- $v_{\text {circ }}$ relation, and

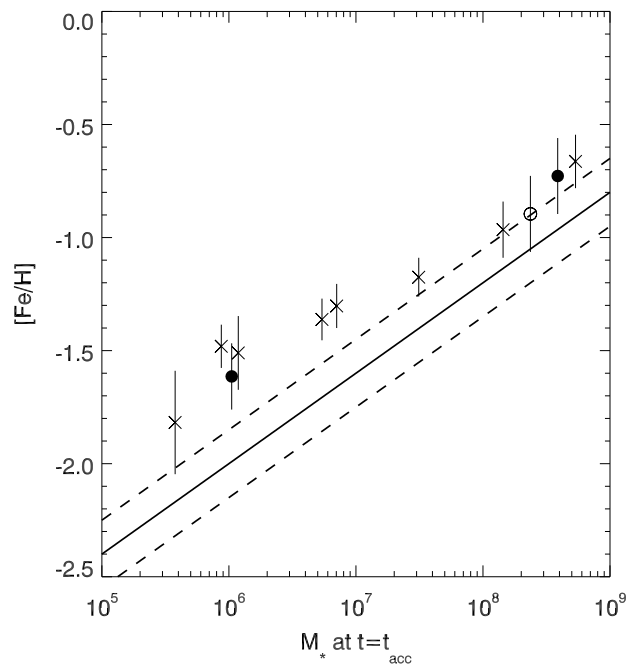

FIG. 2.- The stellar mass - metallicity relation for local dwarf galaxies. We plot the observed trend from Dekel \& Wod (2003) (solid line), the region containing $70 \%$ of the observed data (dashed lines), and the calculated relation for a range in mass of model dwarf galaxies (crosses). The error bars on the model calculations delineate the $\sim 1-\sigma$ metallicity range $(68 \%)$ of stars in each system. We use this calibration to constrain the strength of stellar feedback in our model. We also plot our models for surviving dSph and dIrr galaxies (filled circles) and the halo-progenitor model (open circle) for comparison.

$w_{\mathrm{Fe}}$ by the approximate stellar mass-metallicity relation. Finally, the parameter $w_{\alpha}$ is chosen to reproduce observations of the chemical abundance pattern of dSph type galaxies once the above list of complimentary observational data is invoked to constrain our other free parameters. In the next section we show that our choices for the wind efficiencies indeed reproduce observations of the chemical abundance pattern of dSph-type galaxies. We argue below that the chemical content of low-mass dwarf galaxies should then show trends as a function of stellar mass and circular velocity. Specifically, we expect that high-mass systems will tend to have near solar abundance patterns, while low-mass dwarfs will have intermediate $[\alpha / \mathrm{Fe}]$ ratios.

\section{RESULTS}

The three panels in Figure 3 show the $[\alpha / \mathrm{Fe}]$ vs. $[\mathrm{Fe} / \mathrm{H}]$ tracks computed for our stellar halo progenitor (top), dSph example (middle), and dIrr example (bottom). The tracks correspond to the star formation histories presented in Figure 1 In all cases, solid lines show $[\mathrm{O} / \mathrm{Fe}]$ and dotted lines show $[\mathrm{Mg} / \mathrm{Fe}]$. The final stellar masses (at accretion) for the systems are indicated in the bottom left of each panel. The thick region along each line corresponds to the abundance region inhabited by the middle $68 \%$ of stars in each system (the $68 \%$ spread by mass about the median).

In the upper panel, our massive $\left(M_{0} \simeq 5 \times 10^{10} M_{\odot}\right)$, earlyforming, stellar halo progenitor forms stars quickly until it is accreted into the Galactic halo $\sim 9 \mathrm{Gyr}$ ago. Its gas enriches to $[\mathrm{Fe} / \mathrm{H}]=-0.7$ and an $\alpha$-abundance of $[\alpha / \mathrm{Fe}]=0.1$ just before accretion, while its stars typically have a metallicity of $[\mathrm{Fe} / \mathrm{H}] \simeq-0.9$ and $[\alpha / \mathrm{Fe}] \simeq 0.2$. Upon accretion, we expect this system to quickly disrupt, and contribute its stars to the stellar halo $(\S 2)$. Over the predicted chemical evolution tracks we plot abundance data (crosses) for galactic halo stars from Table 2 of Venn et al. (2004), from which we select all stars 


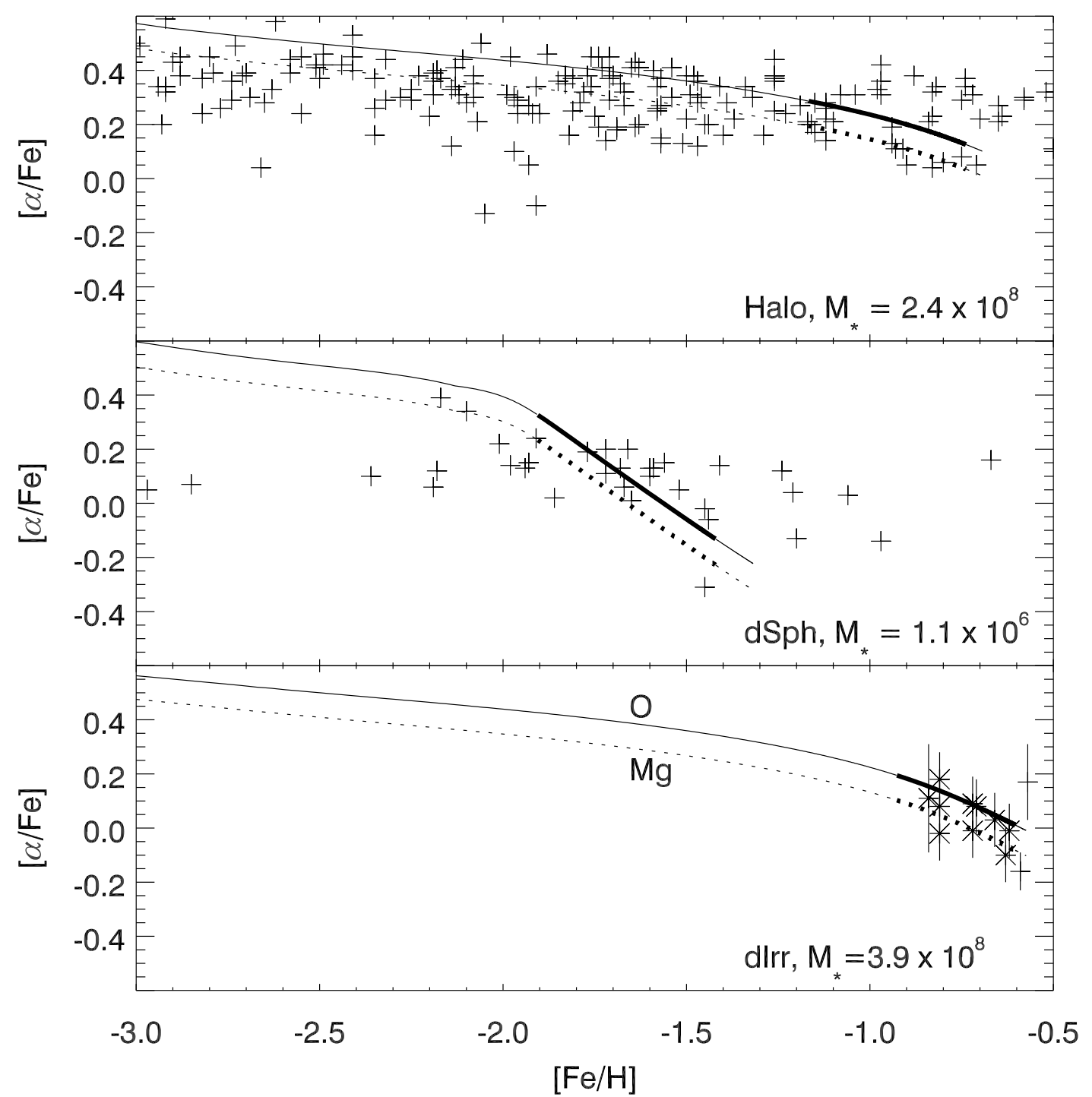

FIG. 3.- The $[\alpha / \mathrm{Fe}]$ vs. [Fe/H] abundance of our halo-progenitor (top panel), dwarf spheroidal (middle panel), and dIrr-type galaxy (lower panel). Top: The halo-progenitor model retains a super-solar $[\alpha / \mathrm{Fe}]$ abundance pattern, resembling the stellar halo data (crosses, from Venn et al. 2004). Middle: The abundance pattern of the dwarf spheroidal galaxy is set by the strength of the stellar feedback, which forces its $[\alpha / \mathrm{Fe}]$ abundance below that of halo stars. For comparison, we overplot abundance data of similarly-sized dSph galaxies from Venn et al. (2004) as crosses. Bottom: The majority of the star formation in the massive dIrr occurs at near-solar values of $[\alpha / \mathrm{Fe}]$ and at higher metallicities, similar to stellar abundances in the SMC (stars and crosses from Venn 1999; Hill et al. 1995, respectively). Note that we expect extremely metal poor stars in the SMC and LMC to have enhanced, halo-like $[\alpha / \mathrm{Fe}]$ ratios.

with Galactic halo membership probabilities greater than $50 \%$ 5 . The halo-progenitor dwarf galaxy abundance pattern is consistent with the overall abundance pattern of the Galactic halo. Agreement between our model and the data arises because the stellar halo progenitor is accreted and destroyed before significant Type I enrichment can occur. More generally, we expect that the super-solar $\alpha$-abundance of halo stars is inherited naturally from the star formation and chemical enrichment histories of these massive, early-accreted dwarf galaxies (Font et al. 2005, in preparation). As discussed in \$2, the $\sim 1.5 \times 10^{9} M_{\odot}$ stellar mass of the halo (Carnev et al. 1990)

5 The data was originally obtained by Fulbright 2000, 2002); Gratton \& Sneden (1988, 1991, 1994); Hanson et al. (1998); Ivans et al. (2003); McWilliam et al. (1995); McWilliam (1998); Rvan et al. (1996); Stephens \& Boesgaard (2002). is expected to be dominated by stars from disrupted systems of this type (Bullock \& Johnston 2005).

In the middle panel of Figure 3 our low-mass $\left(M_{0} \simeq\right.$ $\left.5 \times 10^{8} M_{\odot}\right)$, dSph example forms stars more gradually, and is accreted onto the Milky Way halo $\sim 5$ Gyr ago. Metal enrichment is suppressed in this system compared to the other two panels because the dSph halo is more loosely bound and supernovae winds can more easily escape $(\S 4.2)$. Since the overall ejection rate is higher, the relative efficiency of blowout for Type I and Type II supernovae ejecta is more apparent and creates the break in the trajectory in the dSph track. For comparison, the crosses show $\alpha$-abundance data compiled by Venn et al. (2004) for Local Group dwarf spheroidal galaxies. The data reflects the well-established disparity between the $[\alpha / \mathrm{Fe}] \sim 0.3$ abundance ratios of Galactic stellar halo stars and the $[\alpha / \mathrm{Fe}] \sim 0.0-0.2$ abundance ratios of lo- 
cal, low-mass dwarf galaxies at similar metallicities. It is encouraging that our model $\mathrm{dSph}$ evolves on an $\alpha$-abundance track that overlaps with data of observed dwarf spheroidal galaxies, with its final stellar abundance peaking at $[\alpha / \mathrm{Fe}] \simeq$ 0.05 and $[\mathrm{Fe} / \mathrm{H}] \simeq-1.65$. We emphasize again that this agreement stems from supernovae blow-out. The differences between the $\alpha$-abundance pattern for our halo progenitor and our dSph example primarily results from the relative potential well depths of their host dark matter halos. However, we note that differences between the $\alpha$-abundance pattern of specific dSph galaxies may reflect different efficiencies for blowout of Type Ia and Type II supernovae ejecta between systems (c.f. Lanfranchi \& Matteucci 2004) than what we adopt above. In principle, differences in blow-out efficiencies between systems will depend on the complicated hydrodynamical problem of following the overlap of supernovae bubbles and cannot be properly addressed by our simple modelling. Similarly, our choice of initial mass function affects the number of supernovae per unit mass of stars formed, but such alterations to our modeling can be accomodated through our feedback calibration and similar results can be obtained for e.g. the Salpeter (1955) IMF. We therefore rely on the observed trends of $\alpha$-abundance in dSph galaxies, the DekelWoo relation, the total stellar mass of the Galactic halo, and the stellar mass- $v_{\text {circ }}$ relation expected for Local Group dwarfs to constrain our singular choices for the blow-out efficiencies that we utilize in our modelling.

Finally, the $[\alpha / \mathrm{Fe}]$ vs $[\mathrm{Fe} / \mathrm{H}]$ track for our massive $\left(M_{0} \simeq\right.$ $5 \times 10^{10} M_{\odot}$ ) dIrr example evolves in a similar manner to that of our stellar halo progenitor case, except that it is accreted much later and can continue evolving for several more billion years. Because of this longer, slower star formation history, the effect of Type I supernovae is more pronounced than in the halo-progenitor case. The gas metallicity enriches to $[\mathrm{Fe} / \mathrm{H}]$ $=-0.6$ with an $\alpha$-abundance of roughly solar, while the inner $68 \%$ of stars by mass span a metallicity of $[\mathrm{Fe} / \mathrm{H}]=-0.95$ to $[\mathrm{Fe} / \mathrm{H}]=-0.6$ and range from $[\alpha / \mathrm{Fe}]=0.2$ to near solar values. Indeed, it has abundance patterns typical of massive satellite galaxies in the local group, as illustrated by the overplotted from LMC stars by Hill et al. (1995) and SMC stars by Venn (1999). Note that we predict that the lowest metallicity stars in galaxies like the SMC and LMC should have abundance patterns similar to those in halo stars. The similarity of $\alpha$-abundance patterns in dwarf galaxies at early times suggests we cannot rule out a scenario in which the Milky Way stellar halo was completely comprised from the destruction of dSph galaxies before they were significantly enriched by Type I supernovae. However, the statistics of dark matter halo accretion histories (see Zentner et al. 2005) suggest such a scenario is unlikely in the context of hierarchical formation of the stellar halo. Furthermore, dynamical modelling of the formation of the stellar halo might rule out such a scenario through differences in the resultant spectrum of phase-space correlations.

Overall, the differences in the shape of the abundance pattern tracks between the halo, dSph, and dIrr models are straightforwardly attributable to the mass-dependent strength of the feedback implementation. The stronger winds in the low-mass dSph model cause the $[\alpha / \mathrm{Fe}]$ abundance to drop near $[\mathrm{Fe} / \mathrm{H}]=-2$ as $\sim 70 \%$ of the metals produced by star formation have been expelled from the system by this time. At the same metallicity, the halo progenitor and dIrr models have only lost $\sim 7 \%$ and $\sim 9 \%$ of their metals, respectively. For the $\mathrm{dSph}$ model, Type I SN begin to dominate over Type II SN by this metallicity and the retention of Fe relative to $\alpha$-elements correspondingly increases. The relative strength of the $\alpha$ - and Fe-wind efficiencies will vary between individual dSph galaxies (see, e.g., Lanfranchi \& Matteucci 2004) to produce their various abundance patterns, but the $[\alpha / \mathrm{Fe}]$ tracks of the more massive halo progenitor and dIrr systems will be fairly insensitive to these details as their less efficient winds begin to strongly change their abundance patterns at characteristically higher metallicities.

\section{CONCLUSIONS}

By combining cosmologically-motivated star formation and gas accretion histories for dwarf galaxies with modelling of the chemical yields from stellar populations, we demonstrate that the abundance patterns of the Galactic halo, low-mass dwarf spheroidals, and more massive dwarf irregular galaxies can be understood within the context of cosmologicallymotivated models of the stellar halo and dwarf galaxy formation. A straightforward prediction of $\Lambda \mathrm{CDM}$ is that most of the stars in the stellar halo were formed in dIrr-sized dark matter halos that were accreted at early times and subsequently destroyed. The destroyed, proto-halo systems are short-lived, form their stars rapidly, and are enriched predominately by Type II supernovae. They are disrupted before the iron input from Type I supernovae can drive them to higher metallicities, leaving a chemical abundance pattern that matches observations of low-metallicity, $\alpha$-enriched halo stars.

Longer-lived, dIrr-type galaxies that survive to the present day, form their stars more gradually. These systems undergo both Type I and Type II supernovae enrichment. We presented a "typical" dIrr-type accretion history, which enriched to a moderate metallicity with an abundance pattern similar to that of the SMC and LMC. We predict that lower-metallicity stars in the SMC or LMC will likely show similar abundance patterns to those of halo stars.

Low-mass dSph-type galaxies are likely systems whose chemical abundance patterns have been altered significantly by supernovae winds, as their shallow potential wells cannot retain outflows. Adopting simple assumptions about the ejection wind efficiency, we showed that the expected population of surviving dwarf satellite galaxies in $\Lambda$ CDM galactic halos match the stellar-mass/metallicity relation observed for localgroup dwarfs (Dekel \& Woo 2003). We then argued that the same stellar feedback is essential in explaining the $\alpha$-element abundance patterns in $\mathrm{dSph}$ galaxies, and demonstrated this idea by presenting an explicit, cosmologically-motived dSph model that follows the abundance pattern of the observed dwarf spheroidals successfully. Our expectation is that the abundance data for dwarf spheroidal galaxies as a function of their stellar mass can be matched by similar, cosmologicallymotivated star formation histories (Font et al., in preparation). We predict that $[\alpha / \mathrm{Fe}]$ ratios should gradually approach the solar value in dwarf galaxies of increasing stellar mass (or velocity dispersion).

As discussed, most of the stars in the Galactic stellar halo are likely remnants of early-forming dIrr-type galaxies that chemically bear little resemblance to present-day dwarf spheroidals. At the same time we expect that a significant number of dSph-size galaxies have been accreted and destroyed over the Milky Way's history. Because of their shallow potential well depths, these low-mass dwarf remnants should be chemically distinct from most of the background population of the stellar halo. Fossil evidence of these accretion events may be more easily detected if chemical signatures 
are used to help identify substructure (e.g. Nissen \& Schuster 1997; Font et al. 2005, in preparation). We also note that further constraints on our modeling are supplied by observations of the age and metallicity distribution of halo stars. While the modeling presented here demonstrates that the chemical abundance pattern of typical halo progenitors match the observed $[\alpha / \mathrm{Fe}]$ ratio in the Galactic halo, our model must be extended account for the full ensemble of halo progenitors expected from the time-dependent mass spectrum of accreted substructure predicted by the $\Lambda$ CDM scenario for structure formation. Such an improvement to our modeling will be presented in future work (Font et al. 2005, in preparation).

While we adopt a feedback formulation commonly used to model chemical evolution in galaxies, we note other feedback models may be more physically motivated. For instance, hydrodynamical simulations of galaxy formation may include multiphase descriptions of the interstellar medium (McKee \& Ostriker 1977) that may more naturally produce self-consistent star formation histories for dwarf galaxies as the microphysics of the ISM are better captured (e.g. Springel \& Hernquist 2003). Hydrodynamic simulations can also include the effects of supernovae-driven galactic winds formulated in a manner similar to what we employ, but the detailed calculations of the expansion and cooling of shocks allow a reduction in the net number of free parameters (for instance the need for $\epsilon$ is eliminated, see Springel \& Hernquist 2003). As these forms of feedback affect the star formation history and gas content of a given object, they will also affect galactic chemical evolution. We also note it is possible that some dwarf galaxies with spheroidal components contain intermediate-mass supermassive black holes (Barth et al. 2004; Greene \& Ho 2004), and hydrodynamical simulations of mergers of galaxies containing supermassive black holes with thermal feedback driven by Bondi (1952)-style accretion (Springel et al. 2004; Di Matteo et al. 2005) demonstrate that supermassive black holes may have considerable impact on the star formation history and gas content of their host systems. Feedback from supermassive black holes may then significantly alter the chemical evolution history of galaxies in a manner not considered here. Due to the complex hydrody- namical and radiative cooling issues posed by supernovae and black hole feedback, we suggest self-consistent hydrodynamical simulations that include the relevant physics described above may be necessary to model more completely the effects of feedback on the chemical evolution of dwarf galaxies and the Galactic stellar halo. However, to demonstrate the feasibility of a cosmologically-motivated explanation for the abundance of the stellar halo we believe our comparatively simple model is sufficient. We note that our chemical enrichment modeling could be incorporated into the calculations of Bullock \& Johnston (2005) to form a more complete framework to model the detailed properties of the Milky Way stellar halo. We plan to present this model in a future paper (Font et al. 2005, in preparation).

We close by mentioning that our model does not capture the full complexity of the available observations, notably of heavy elements like Ba, Y, and Eu (Venn et al. 2004), and future inconsistencies between the scenario for the formation of the halo presented here and observations may arise. Future modelling could conceivably help draw stronger connections between the very detailed stellar observation and cosmological scenarios for the origin of dwarf galaxies and the stellar halo. For instance, as full chemical modeling becomes incorporated into cosmological simulations of galaxy formation, questions about the chemical abundance of thick disk stars and accreted dwarf systems (c.f. Wyse 2004) will find more satisfying answers (see e.g., Brook et al. 2004b). We therefore encourage future attempts to coordinate detailed chemical modelling with cosmological calculations to increase the interface between cosmology and galactic astronomy.

The authors acknowledge fruitful discussions with K. Venn, M. Shetrone, Q. Y. Gong, A. Zentner, and R. Wyse. This work was supported in part by NSF grants ACI 96-19019, AST 0071019, AST 02-06299, and AST 03-07690, and NASA ATP grants NAG5-12140, NAG5-13292, and NAG5-13381. K.V.J. and A.F.'s contributions were supported through NASA grant NAG5-9064 and NSF CAREER award AST-0133617.

\section{REFERENCES}

Abadi, M. G., Navarro, J. F., Steinmetz, M., \& Eke, V. R. 2003, ApJ, 591, 499

Alam, S. M. K., Bullock, J. S., \& Weinberg, D. H. 2002, ApJ, 572, 34

Barth, A. J., Ho, L. C., Rutledge, R. E., \& Sargent, W. L. W. 2004, ApJ, 607, 90

Bell, E. F., McIntosh, D. H., Katz, N., \& Weinberg, M. D. 2003, ApJ, 585, L117

Blakeslee, J. P., et al. 2003, ApJ, 589, 693

Bondi, H. 1952, MNRAS, 112, 195

Brook, C. B., Kawata, D., Gibson, B. K., \& Flynn, C. 2004, MNRAS, 349, 52

Brook, C. B., Kawata, D., Gibson, B. K., \& Freeman, K. C. 2004, ApJ, 612, 894

Brodie, J. P. \& Huchra, J. P. 1991, ApJ, 379, 157

Brown, W. R., Geller, M. J., Kenyon, S. J., Beers, T. C., Kurtz, M. J., \& Roll, J. B. 2004, AJ, 127, 1555

Johnston, K. V., \& Bullock, J. S. 2005, ApJ, submitted

Bullock, J. S., Kravtsov, A. V., \& Weinberg, D. H. 2000, ApJ, 539, 517

-. 2001, ApJ, 548, 33

Carney, B. W., Latham, D. W., \& Laird, J. B. 1990, AJ, 99, 572

Chiappini, C., Romano, D., \& Matteucci, F. 2003, MNRAS, 339, 63

Cole, A. A., Smecker-Hane, T. A., \& Gallagher, J. S. 2000, AJ, 120, 1808

Colín, P., Avila-Reese, V., \& Valenzuela, O. 2000, ApJ, 542, 622

Dekel, A., \& Silk, J. 1986, ApJ, 303, 39

Dekel, A., \& Woo, J. 2003, MNRAS, 344, 1131

Devriendt, J. E. G., Guiderdoni, B., \& Sadat, R. 1999, A\&A, 350, 381
Di Matteo, T., Springel, V., \& Hernquist, L. 2005, Nature, 433, 604

Ferreras, I., \& Silk, J. 2000, ApJ, 532, 193

Ferrini, F., Matteucci, F., Pardi, C., \& Penco, U. 1992, ApJ, 387, 138

Font, A., Bullock, J. S., Johnston, K. V., \& Robertson, B. 2005, in preparation Fulbright, J. P. 2000, AJ, 120, 1841

-. 2002, AJ, 123, 404

Geisler, D., Smith, V. V., Wallerstein, G., Gonzalez, G., \& Charbonnel, C. 2005, AJ, 129, 1428

Gilmore, G., \& Wyse, R. F. G. 1998, AJ, 116, 748

Gratton, R. G., \& Sneden, C. 1988, A\&A, 204, 193

-. 1991, A\&A, 241, 501

-. 1994, A\&A, 287, 927

Grebel, E. K. 2001, in Dwarf galaxies and their environment, ed. K. S.

De Boer, R. Dettmar, \& U. Klein (Aachen: Shaker Verlag), 45

Grebel, E. K., Gallagher, J. S. \& Harbeck, D. 2003, AJ, 125, 1926

Greene, J. E., \& Ho, L. C. 2004, ApJ, 610, 722

Greggio, L., \& Renzini, A. 1983, A\&A, 118, 217

Grevesse, N., \& Sauval, A. J. 1998, Space Science Reviews, 85, 161

Hanson, R. B., Sneden, C., Kraft, R. P., \& Fulbright, J. 1998, AJ, 116, 1286

Hartwick, F. D. A. 1976, ApJ, 209, 418

Helmi, A., \& White, S. D. M. 1999, MNRAS, 307, 495

Hill, V. 1997, A\&A, 324, 435

Hill, V., Andrievsky, S., \& Spite, M. 1995, A\&A, 293, 347

Hill, V., Barbuy, B., \& Spite, M. 1997, A\&A, 323, 461

Hill, V., François, P., Spite, M., Primas, F., \& Spite, F. 2000, A\&A, 364, L19 
Ivans, I. I., Sneden, C., James, C. R., Preston, G. W., Fulbright, J. P., Höflich, P. A., Carney, B. W., \& Wheeler, J. C. 2003, ApJ, 592, 906

Jasniewicz, G., \& Thevenin, F. 1994, A\&A, 282, 717

Johnston, K. V., Hernquist, L., \& Bolte, M. 1996, ApJ, 465, 278

Kaplinghat, M., Knox, L., \& Turner, M. S. 2000, Physical Review Letters, 85,3335

Kinman, T. D., Saha, A., \& Pier, J. R. 2004, ApJ, 605, L25

Klypin, A., Kravtsov, A. V., Valenzuela, O., \& Prada, F. 1999, ApJ, 522, 82

Korn, A. J., Keller, S. C., Kaufer, A., Langer, N., Przybilla, N., Stahl, O., \&

Wolf, B. 2002, A\&A, 385, 143

Kroupa, P. 2001, MNRAS, 322, 231

Kroupa, P. 2002, Science, 295, 82

Lacey, C., \& Cole, S. 1993, MNRAS, 262, 627

Lahav, O., et al. 2002, MNRAS, 333, 961

Lanfranchi, G. A., \& Matteucci, F. 2004, MNRAS, 351, 1338

Larson, R. B. 1972, Nature Physical Science, 236, 7

Mac Low, M., \& Ferrara, A. 1999, ApJ, 513, 142

Majewski, S. R., Ostheimer, J. C., Rocha-Pinto, H. J., Patterson, R. J., Guhathakurta, P., \& Reitzel, D. 2004, ApJ, 615, 738

Maller, A. H., \& Bullock, J. S. 2004, MNRAS, 355, 694

Mastropietro, C., Moore, B., Mayer, L., Wadsley, J., \& Stadel, J. 2004 preprint (astro-ph/0412312,

Mateo, M. L. 1998, ARA\&A, 36, 435

Matteucci, F., \& Francois, P. 1989, MNRAS, 239, 885

Matteucci, F., \& Greggio, L. 1986, A\&A, 154, 279

Matteucci, F., \& Tornambe, A. 1987, A\&A, 185, 51

McKee, C. F., \& Ostriker, J. P. 1977, ApJ, 218, 148

McWilliam, A. 1998, AJ, 115, 1640

McWilliam, A., Preston, G. W., Sneden, C., \& Searle, L. 1995, AJ, 109, 2736

Moore, B., \& Davis, M. 1994, MNRAS, 270, 209

Moore, B., Ghigna, S., Governato, F., Lake, G., Quinn, T., Stadel, J., \& Tozzi, P. 1999, ApJ, 524, L19

Morrison, H. L., et al. 2003, AJ, 125, 2502

Navarro, J. F., \& Steinmetz, M. 1997, ApJ, 478, 13

Newberg, H. J., et al. 2002, ApJ, 569, 245

Nissen, P. E., \& Schuster, W. J. 1997, A\&A, 326, 751

Nomoto, K., Iwamoto, K., Nakasato, N., Thielemann, F.-K., Brachwitz, F., Tsujimoto, T., Kubo, Y., \& Kishimoto, N. 1997, Nuclear Physics A, 621, 467

Nomoto, K., Thielemann, F.-K., \& Yokoi, K. 1984, ApJ, 286, 644

Pagel, B. E. J., \& Patchett, B. E. 1975, MNRAS, 172, 13

Pen, U., Zhang, T., van Waerbeke, L., Mellier, Y., Zhang, P., \& Dubinski, J. 2003, ApJ, 592, 664

Robertson, B., Yoshida, N., Springel, V., \& Hernquist, L. 2004, ApJ, 606, 32

Ryan, S. G., Norris, J. E., \& Beers, T. C. 1996, ApJ, 471, 254

Salpeter, E. E. 1955, ApJ, 121, 161

Schaller, G., Schaerer, D., Meynet, G., \& Maeder, A. 1992, A\&AS, 96, 269

Searle, L., \& Zinn, R. 1978, ApJ, 225, 357
Shetrone, M., Venn, K. A., Tolstoy, E., Primas, F., Hill, V., \& Kaufer, A. 2003, AJ, 125, 684

Shetrone, M. D., Côté, P., \& Sargent, W. L. W. 2001, ApJ, 548, 592

Simon, J. D., Bolatto, A. D., Leroy, A., Blitz, L., \& Gates, E. L. 2005, ApJ, 621,757

Smecker-Hane, T., \& McWilliam, A. 1999, in ASP Conf. Ser. 192: Spectrophotometric Dating of Stars and Galaxies, ed. I. Hubeny, S. R. Heap, \& R. H. Cornett (San Francisco: ASP), 150

Smecker-Hane, T., \& McWilliam, A. 2003, preprint astro-ph/0205411

Smith, V. V., et al. 2002, AJ, 124, 3241

Spergel, D. N., \& Steinhardt, P. J. 2000, Physical Review Letters, 84, 3760

Spergel, D. N., et al. 2003, ApJS, 148, 175

Springel, V., Di Matteo, T., \& Hernquist, L. 2004, MNRAS, submitted

Springel, V., \& Hernquist, L. 2003, MNRAS, 339, 289

Stephens, A., \& Boesgaard, A. M. 2002, AJ, 123, 1647

Talbot, R. J., \& Arnett, W. D. 1973, ApJ, 186, 51

Tammann, G. A. 1982, in NATO ASIC Proc. 90: Supernovae: A Survey of Current Research, ed. M. J. Rees \& R. J. Stoneham (Dordrecht: D. Reidel), 371

Thielemann, F., Nomoto, K., \& Hashimoto, M. 1996, ApJ, 460, 408

Timmes, F. X., Woosley, S. E., \& Weaver, T. A. 1995, ApJS, 98, 617

Tinsley, B. M. 1972, A\&A, 20, 383

-. 1980, Fundamentals of Cosmic Physics, 5, 287

Tolstoy, E., Venn, K. A., Shetrone, M., Primas, F., Hill, V., Kaufer, A., \& Szeifert, T. 2003, AJ, 125, 707

Tsujimoto, T., Nomoto, K., Yoshii, Y., Hashimoto, M., Yanagida, S., \& Thielemann, F.-K. 1995, MNRAS, 277, 945

Twarog, B. A. 1980, ApJ, 242, 242

Unavane, M., Wyse, R. F. G., \& Gilmore, G. 1996, MNRAS, 278, 727

van den Bergh, S. 1962, AJ, 67, 486

van den Hoek, L. B., \& Groenewegen, M. A. T. 1997, A\&AS, 123, 305

van der Marel, R. P., Alves, D. R., Hardy, E., \& Suntzeff, N. B. 2002, AJ, 124,2639

Venn, K. A. 1999, ApJ, 518, 405

Venn, K. A., Irwin, M., Shetrone, M. D., Tout, C. A., Hill, V., \& Tolstoy, E. 2004, AJ, 128, 1177

Wechsler, R. H., Bullock, J. S., Primack, J. R., Kravtsov, A. V., \& Dekel, A. 2002, ApJ, 568, 52

Wheeler, J. C., Sneden, C., \& Truran, J. W. 1989, ARA\&A, 27, 279

Wyse, R. F. G. 2004, in The Local Group as an Astrophysical Laboratory (Cambridge: Cambridge University Press)

Zehavi, I., et al. 2002, ApJ, 571, 172

Zaritsky, D. and Kennicutt, R. C. and Huchra, J. P. 1994, ApJ, 420, 87

Zentner, A. R., Berlind, A. A., Bullock, J. S., Kravtsov, A. V., \& Wechsler, R. H. 2005, ApJ, 624, 505

Zentner, A. R., \& Bullock, J. S. 2003, ApJ, 598, 49 